\title{
Electromechanical response at polar zigzag boundaries in hybrid monolayers
}

\author{
Rafael Martinez-Gordillo and Miguel Pruneda* \\ ICN2-Institut Catala de Nanociencia i Nanotecnologia, and CSIC-Consejo Superior de Investigaciones Cientificas, ICN2 Building, \\ Campus UAB, 08193 Bellaterra (Barcelona), Spain
}

(Received 19 November 2014; published 9 January 2015)

\begin{abstract}
First-principles calculations are used to demonstrate electromechanical control of charge and spin at zigzagedged interfaces between graphene and boron-nitride domains in hybrid monolayers. We show how, through a direct piezoelectric effect, the interfacial bound charges and associated electric fields can be tuned by application of an external mechanical force (stress) on the system. This results in mechanical control of the edge magnetization (piezomagnetic effect), and the possibility to transform a semiconducting heterostructure into a half-metal. The inverse effect (application of an external electric field to induce a mechanical deformation) goes together with a magnetoelectric response, which under ideal conditions we estimate to be comparable to that of prototypical $\mathrm{Cr}_{2} \mathrm{O}_{3}$. These effects originate from the magnetic properties of graphene's zigzag edges and the dielectric properties of the boron-nitride domain, and can also be expected in any other coplanar heterostructures with polar discontinuities.
\end{abstract}

DOI: 10.1103/PhysRevB.91.045411

PACS number(s): 73.20.-r, 75.75.-c, 77.55.-g, 77.65.-j

\section{INTRODUCTION}

Hybrid C/BN nanostructures offer a new route to engineering electronic and optical properties of graphene-based devices. Following the impressive advances in the experimental growth of these nanostructures [1-5], the physical properties of coplanar domain-segregated $\mathrm{C}$ and $h$-BN nanosheets and nanotubes are being intensively studied. High-resolution scanning tunneling microscopy images showed that zigzag interfaces are preferably formed [3,5]. A number of very recent experiments have observed that in these zigzag boundaries between graphene and $h$-BN domains, novel interfacial electronic states appear [6-8], thus confirming early theoretical predictions [9-16].

Both graphene and BN have remarkable mechanical strength and flexibility, and are able to sustain huge elastic structural deformations. Although the strain modulation of band gaps has been studied before [17,18], the coupling of mechanical and electrostatic properties in hybrid C/BN nanostructures still needs to be explored. This becomes critical if one takes into account that, for noncentrosymmetric dielectric crystals (such as $h$-BN), application of mechanical strains gives rise to polarization fields and interfacial bound charges. Remarkable piezoelectric and flexoelectric properties in twodimensional $h$-BN have been theoretically predicted [19-21], and pose an alternative route for controlling electronic properties in hybrid $\mathrm{C} / \mathrm{BN}$ heterostructures, as well as other hybrid coplanar materials and polar engineered heterostructures [22].

The polarity of the zigzag edge in $\mathrm{BN}$ domains gives an interfacial dipole, hence an effective electric field acting on the graphene domain in hybrid monolayers. It has been predicted that half-metallicity can be induced in zigzag-edged graphene nanoribbons (GNRs) by application of a sufficiently large in-plane electric field perpendicular to the edges of the ribbon [23], and we can envision that narrow graphene ribbons embedded in coplanar BN can have modified electronic properties. Indeed, several theoretical works have shown that

\footnotetext{
*miguel.pruneda@cin2.es
}

half-metallic properties can be obtained in hybrid $\mathrm{C} / \mathrm{BN}$ heterostructures $[9,13,24,25]$.

Half-metallicity is very appealing for several potential spintronic applications, and, in combination with carbon-based materials, offers an exciting avenue for devices and new discoveries. Typically, in spintronic devices a ferromagnetic electrode is needed to set the spin orientations. However, electric field control of spin transport has important advantages (reduced power consumption, enhanced miniaturization), and magnetoelectric effects in carbon nanostructures are of much interest. In addition to the half-metallicity induced by transversal external electric fields on freestanding GNRs [23], mentioned before, magnetoelectric couplings were predicted for bilayer GNR on silicon substrates by electric bias control of the charge carrier [26], and as a means to favor antiferromagnetic over ferromagnetic configurations in doped ribbons [27]. Similarly, control of magnetism through mechanical deformation would be very valuable for applications. Here we will prove that half-metallicity in hybrid $\mathrm{C} / \mathrm{BN}$ nanostructures can be engineered by magnetoelectric and piezomagnetic effects.

In this paper we use density functional theory calculations to show how the polarity discontinuity at the zigzag-shaped boundaries of $\mathrm{BN}$ can be used to tune the electronic and magnetic properties in hybrid C/BN nanostructures. Direct application of tensile strains modifies the interfacial bound polarization charge and hence changes the population of the electronic edge states which are spin polarized. This results in a piezomagnetic response. Alternatively, the edge states can also be tuned by application of an external electric field, and, in combination with the half-metallicity in hybrid $\mathrm{C} / \mathrm{BN}$ heterostructures, result in a magnetoelectric response, which we evaluate for an ideal composition and geometry to be larger than the one predicted for graphene ribbons on silicon substrates [26].

\section{METHODOLOGY}

As in previous works $[13,15]$, here we consider coplanar geometries composed of two-dimensional superlattices made from $n$ zigzag chains of graphene and $m$ zigzag chains of 
(a)

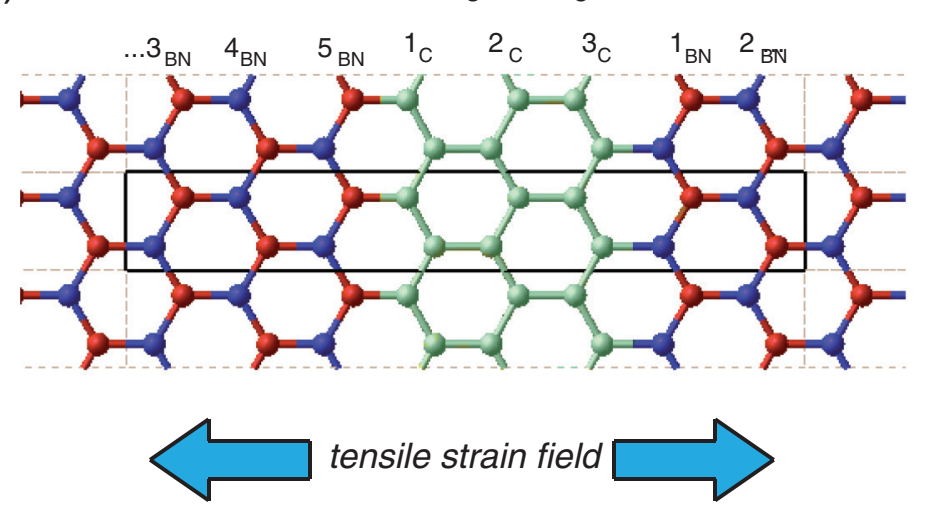

(b)

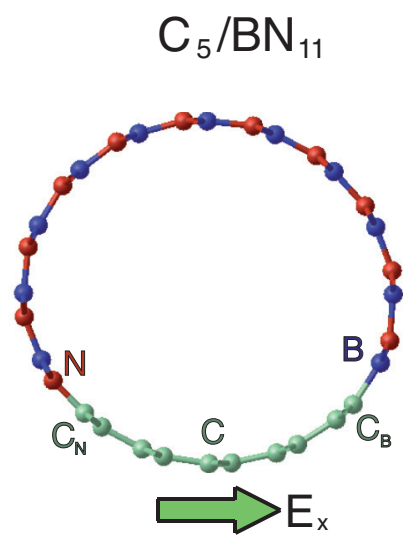

FIG. 1. (Color online) (a) Model geometry for a planar $\mathrm{C}_{3} /(\mathrm{BN})_{5}(n=3, m=5)$ superlattice. The simulation unit cell is shown as a solid line, and periodic images as dashed lines. The numbers of graphene and $h$-BN zigzag chains are marked on top. (b) Geometry for $(8,8)$ hybrid $\mathrm{C} / \mathrm{BN}$ armchair nanotubes, composed of a $\mathrm{C}$ strip with five zigzag chains and a BN strip with 11 zigzag chains along the $z$ axis. Transversal electric fields along the $x$ and $y$ axes are considered in this work.

$\mathrm{BN}$, periodically repeated along the interfacial direction. We label these superlattices as $(n, m)$, or, alternatively, $\mathrm{C}_{n}(\mathrm{BN})_{m}$. While large graphene domains give nonmagnetic semimetallic nanosheets, systems with relatively narrow graphene strips $(n \leqslant 8)$ can be either semiconducting (for narrow $\mathrm{BN}$ domains), or show half-metallic states (for sufficiently wide BN domains, $m \geqslant 6$ ) [13]. We study these planar nanostructures with different chemical compositions and domain sizes under uniaxial tensile strains perpendicular to the $\mathrm{C} / \mathrm{BN}$ interfaces [Fig. 1(a)]. Strains are imposed by fixing the superlattice periodicity while relaxing the atomic positions and perpendicular lattice parameter. Compressive strains, on the other hand, are likely to result in nonplanar ground-state geometries, where flexoelectric effects [19] could play some role. We explored such a possibility but observed little effect on the electronic band structures, and the results will not be discussed here.

$A b$ initio density functional calculations within the spinpolarized generalized-gradient approximation [28], as implemented in the SIESTA code [29], were performed throughout this work. Troullier-Martin type pseudopotentials [30] and numerical atomic orbitals with double- $\zeta$ plus polarization were used to describe the electronic valence states. An accurate description of the interfacial electronic states required a smooth sampling of the reciprocal space, and typically Monkhorst-Pack grids of at least $1 \times 1 \times 100$ were used to sample the Brillouin zone. The atomic positions were determined with a structural relaxation until the forces became lower than $0.02 \mathrm{~V} / \AA$. To avoid spurious interactions between periodic images, we include a vacuum layer of at least $30 \AA$ along the normal direction to the sheet.

In our systems, the relevant bands close to the Fermi level are the valence $\pi_{\mathrm{B}}$ and conduction $\pi_{\mathrm{N}}^{*}$ bands, which are mainly localized at carbon atoms close to the $\mathrm{B}$ and $\mathrm{N}$ edges, respectively. In the half-metallic phase, these two interfacial states become spin polarized, with antiferromagnetic coupling between each edge due to the intrinsic electrostatic potential induced by the polar BN interface [13]. The polar discontinuity at the boundary between $\mathrm{C}$ and $\mathrm{BN}$ domains gives rise to bound charges at the interface that are partially compensated by free carriers in the graphene ribbon, and in consequence, a charge transfer from the $\pi_{\mathrm{B}}$ state to the $\pi_{\mathrm{N}}^{*}$ state [31]. In the following we will show how the interfacial magnetism can be tuned by application of strain (piezomagnetic effect), or by application of an electric field (magnetoelectric effect).

\section{RESPONSE TO MECHANICAL DEFORMATIONS}

Application of a tensile strain in planar $\mathrm{C} / \mathrm{BN}$ heterostructures increases the effective in-plane polarization of $\mathrm{BN}$ domains and consequently the bound (polarization) charge at the interface. The free carriers in the graphene ribbon compensate this bound charge by increasing the electronic transfer from the $\mathrm{B}$ to $\mathrm{N}$ edges. Figure 2(a) plots the Hirshfeld population analysis for the $\mathrm{C}, \mathrm{B}$, and $\mathrm{N}$ atoms at the $\mathrm{C} / \mathrm{BN}$ interfaces, as a function of the applied uniaxial strain. Nitrogen and boron atoms have negative (excess electrons) and positive (deficit electron) charges, respectively, and their values increase almost linearly with tensile strain due to the direct piezoelectric response in $h$-BN. Our computed piezoelectric coefficient in monolayered $h$-BN, $d_{11}=2.06 \times$ $10^{-10} \mathrm{C} / \mathrm{m}$, is in good agreement with the theoretical values reported by Naumov et al. [17] $\left(2.67 \times 10^{-10} \mathrm{C} / \mathrm{m}\right)$, Duerloo et al. [21] $\left(1.38 \times 10^{-10} \mathrm{C} / \mathrm{m}\right)$, and López-Suárez et al. [32] $\left(3.08 \times 10^{-10} \mathrm{C} / \mathrm{m}\right)$, and allows us to predict the evolution of the total charges at the interface with applied strain. As all the charge redistribution takes place within the localized edge electronic states, the total edge charges can be defined as the sum of the Hirshfeld populations for boron and its closest carbon atom $\left(\mathrm{C}_{\mathrm{B}}\right)$ on one side, and nitrogen and its closest carbon atom $\left(\mathrm{C}_{\mathrm{N}}\right)$ on the other side. As seen in the figure, these total charges (diamond symbols) nicely follow the slope predicted by $d_{11}$ (red dashed lines). Notice that $\mathrm{C}_{\mathrm{B}}\left(\mathrm{C}_{\mathrm{N}}\right)$ is negatively (positively) charged and its net charge decreases for increasing strains, because free electrons in the $\mathrm{C}$ strip are transferred from the $\mathrm{B}$ edge to the $\mathrm{N}$ edge. This corresponds to an increased stabilization of the $\pi_{N}^{*}$ state. Figure 3(a) shows the evolution of the band structure for a half-metallic phase under strain, and how the lowering of the $\pi_{\mathrm{N}}^{*}$ band with $\beta$ spin comes at the expense of the depopulation of the $\pi_{\mathrm{B}}$ band with the same spin. This results in an increase in the edge 

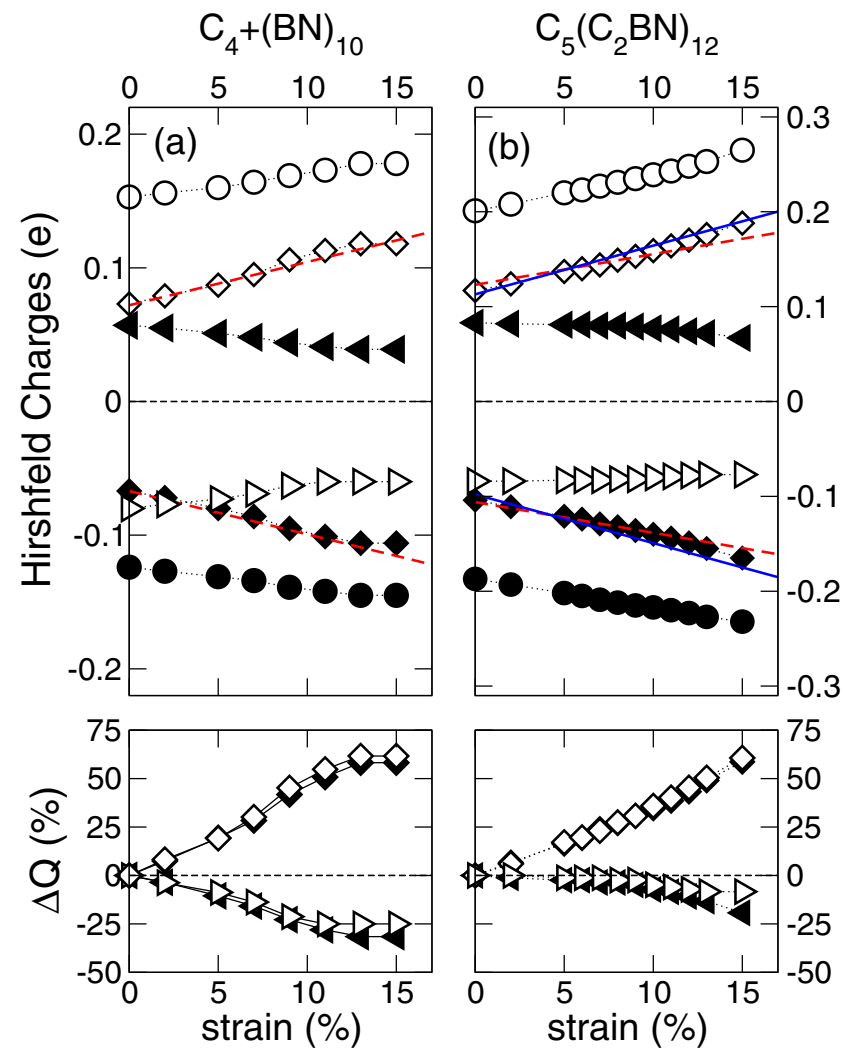

FIG. 2. (Color online) Hirshfeld population analysis for atoms at the boron edge (open) and nitrogen edge (solid symbols), as a function of tensile strain field perpendicular to the interface between domains of graphene and (a) $\mathrm{BN}$ or (b) $\mathrm{BC}_{2} \mathrm{~N}$. Atomic charges for $\mathrm{C}(\mathrm{BN})$ are shown as triangles (circles), while diamonds correspond to the sum of charges of $\mathrm{C}$ and $\mathrm{B}(\mathrm{N})$ at the $\mathrm{C}-\mathrm{B}(\mathrm{C}-\mathrm{N})$ interface. The dashed (solid) lines correspond to the theoretical prediction for the bound charge due to the $\mathrm{BN}\left(\mathrm{BC}_{2} \mathrm{~N}\right)$ polarization. The bottom panel plots the change in the atomic charge at each interface.

magnetization that is characterized in terms of the Mulliken populations on the $\mathrm{C}$ atoms at the $\mathrm{B}$ and $\mathrm{N}$ edges (denoted as $\mathrm{C}_{\mathrm{B}}$ and $\mathrm{C}_{\mathrm{N}}$ ), plotted in Fig. 4. For large strain fields, however, the $\beta$-spin $\pi_{\mathrm{N}}^{*}$ band becomes fully occupied ( $\pi_{\mathrm{B}}$ empty) and a gap opens. At this point, the occupied bands close to the Fermi level are localized at the $\mathrm{N}$ edge for $\beta$ spin and the $\mathrm{B}$ edge for $\alpha$ spin, while the empty bands are B- and N-edge localized (for $\beta$ and $\alpha$ spins, respectively).

Interestingly, one can use strain not only to tune the gaps in systems that are originally half-metallic, but also to induce such a state for systems that, unstrained, are semiconducting. This is illustrated in Figs. 3(b) and 5, where the band gaps for each spin component, as well as the interatomic distances for $\mathrm{C}-\mathrm{N}$ and $\mathrm{C}-\mathrm{B}$ at the edges, are plotted as a function of tensile strain for a variety of chemical compositions. For the semiconducting systems in Figs. 5(a)- 5(c) it is shown how, upon strain, the increased polarization of the BN domain triggers the half-metallic instability in the graphene domain (unshaded white region). Under extreme strain loads, the nanosheet breaks at the C-B junction, as illustrated by the dramatic increase in the interatomic distances (striped region at the right side of each panel). The strain threshold needed
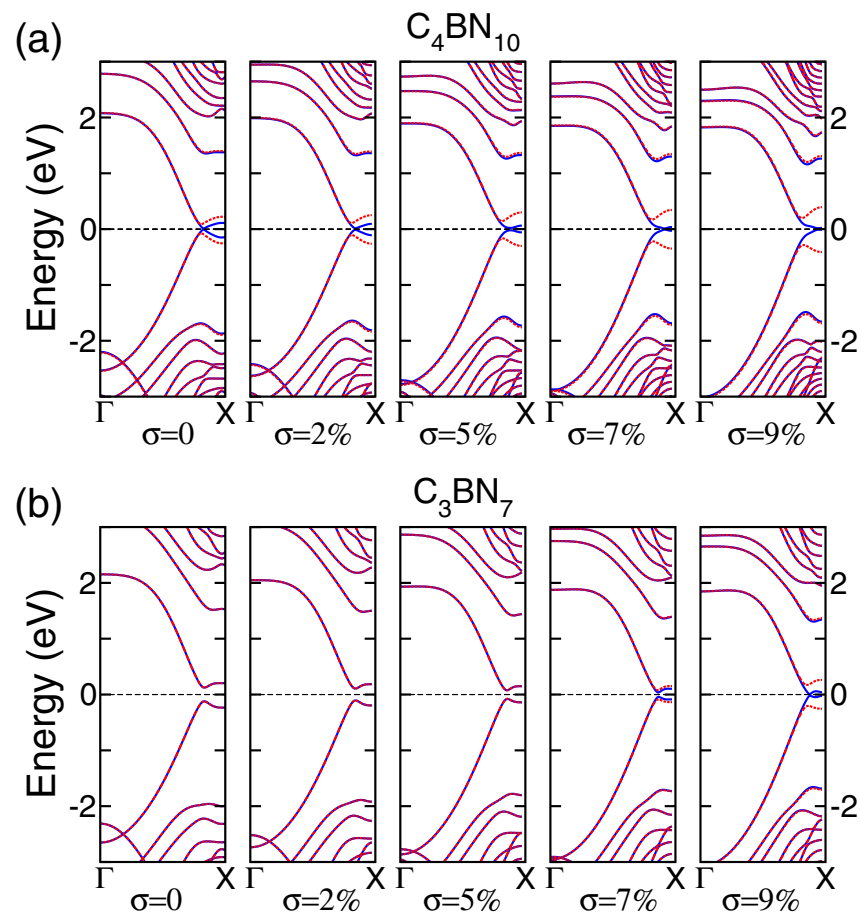

FIG. 3. (Color online) Band structure close to the Fermi level (horizontal dashed line) as a function of uniaxial tensile strain $\sigma$ perpendicular to the $\mathrm{C} / \mathrm{BN}$ interface, for two different widths for the $\mathrm{C}$ and $\mathrm{BN}$ domains: (a) Half-metallic $\mathrm{C}_{4}(\mathrm{BN})_{10}$ and (b) semiconducting $\mathrm{C}_{3}(\mathrm{BN})_{7}$. Red dotted and blue solid lines correspond to $\alpha$ and $\beta$ spins, respectively. Energies are given in $\mathrm{eV}$.

to close the gap depends on the width of both the C and BN ribbons, and so does the strain required to open the gap again from half-metallicity (see the discussion above, and the lower panels). Consequently, there is a range of values for strain under which one could tune half-metallic properties in these hybrid C/BN heterostructures.

Recently, ultrathin graphene nanoribbons segregated from boron-carbon-nitride domains have been experimentally

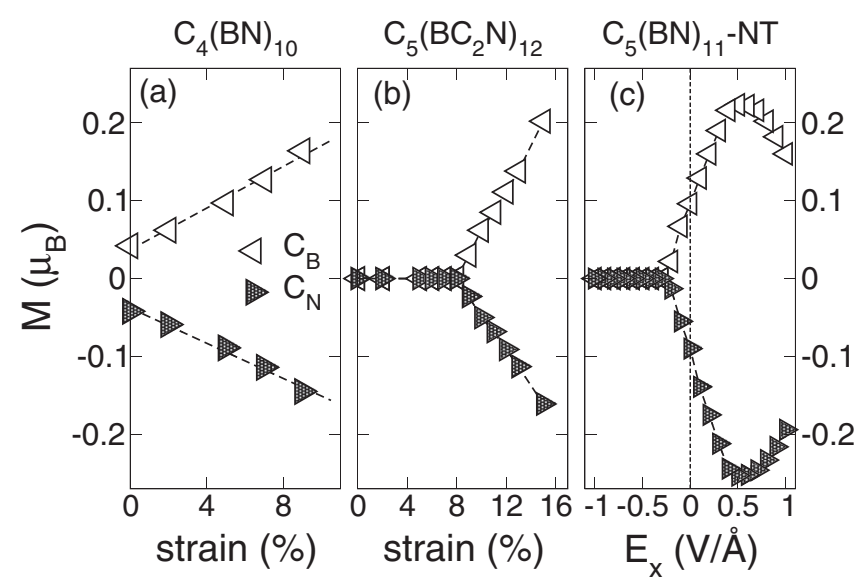

FIG. 4. Evolution of edge magnetization, defined from the Mulliken populations for carbon atoms at the interfaces, as a function of tensile strain for $(\mathrm{a}) \mathrm{a}(4,10) \mathrm{C} / \mathrm{BN}$ superlattice, (b) a $(5,12) \mathrm{C} / \mathrm{BC}_{2} \mathrm{~N}$ superlattice, and (c) a $(5,11) \mathrm{C} / \mathrm{BN}$ armchair nanotube. 

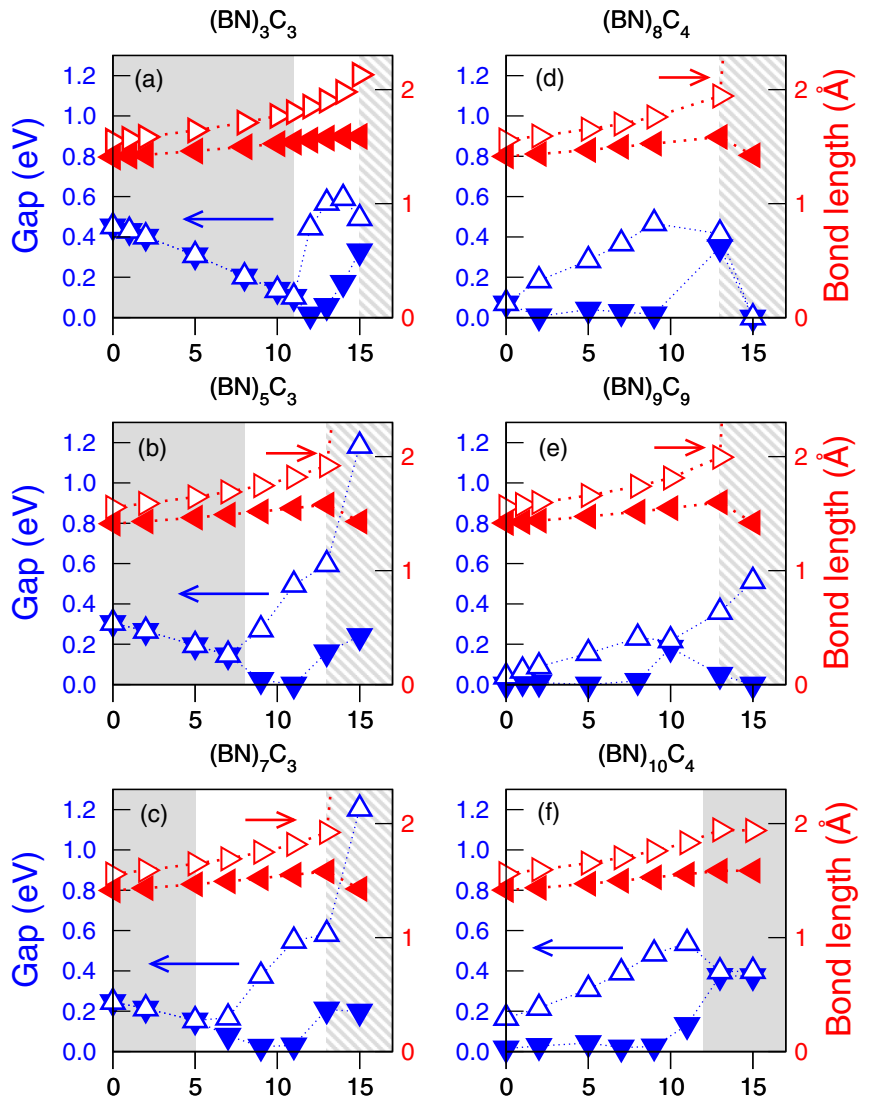

FIG. 5. (Color online) Band gaps for both spin components (up and down triangles) as a function of tensile strain for (a)-(d) semiconducting, (e) semimetallic, and (f) half-metallic C/BN zigzagedged superlattices. Right open and left solid triangles show the C-B and $\mathrm{C}-\mathrm{N}$ bond lengths, respectively. Energy (left) and length (right) scales are in $\mathrm{eV}$ and $\AA$.

observed [33]. Scanning tunneling microscopy imaging found two dominant configurations for stoichiometrically percolated $\mathrm{BC}_{2} \mathrm{~N}$ that correspond to the type I and type II polymorphic structures that were theoretically predicted in the late 1980's [34]. The type II isomer does not have inversion symmetry (or threefold symmetries) and our computed piezoelectric coefficient $\left(d_{11}=3.89 \times 10^{-10} \mathrm{C} / \mathrm{m}\right)$ is larger than that for $\mathrm{BN}$ (in fact, unlike $\mathrm{BN}$, type $\mathrm{II} \mathrm{BC}_{2} \mathrm{~N}$ has a formal in-plane polarization of $0.713 \times 10^{-10} \mathrm{C} / \mathrm{m}$ pointing along the armchair direction). To explore whether half-metallicity could be induced in these hybrid CBN nanostructures, we performed calculations for a variety of geometries with zigzag graphene ribbons embedded in the $\mathrm{BC}_{2} \mathrm{~N}$ domains. We found that strain-induced half-metallicity is possible for $\mathrm{C}$ domains that are broader than five zigzag chains, while the band gaps in ultranarrow graphene ribbons are too large to experience the Zener-breakdown mechanism induced by the polarization discontinuity. The strain evolution of the atomic charges at the interface are again in agreement with the prediction obtained from the piezoelectric response, as shown in Fig. 2(b) for a $(5,12)$ superlattice of $\mathrm{C}$ and $\mathrm{BC}_{2} \mathrm{~N}$. The edge magnetization plotted in Fig. 4(b) reveals the critical strain needed to develop half-metallicity in the system (around $8 \%$ ), and the steep increase in the edge magnetization unravels a piezoantiferromagnetic effect.

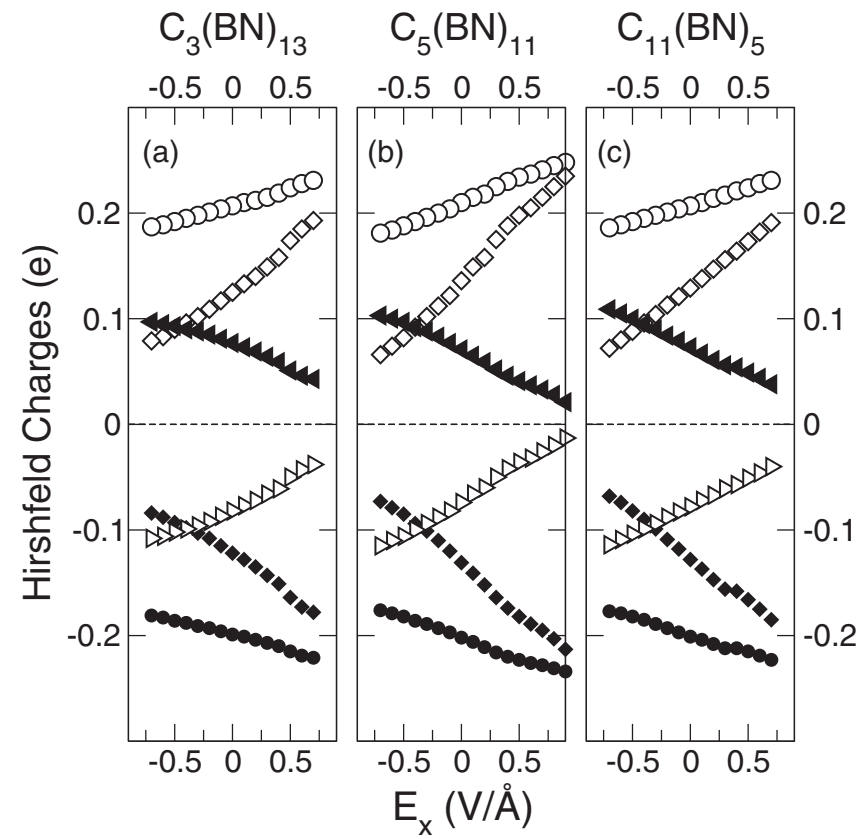

FIG. 6. Hirshfeld population analysis for atoms at the boron edge (open symbols) and nitrogen edge (solid symbols), as a function of applied electric field perpendicular to the interface between domains of graphene and $\mathrm{BN}$ for (a) semiconducting $\mathrm{C}_{3}(\mathrm{BN})_{13}$, (b) halfmetallic $\mathrm{C}_{5}(\mathrm{BN})_{11}$, and semimetallic $\mathrm{C}_{11}(\mathrm{BN})_{5}$. Atomic charges for $\mathrm{C}(\mathrm{BN})$ are shown as triangles (circles), while diamonds correspond to the sum of charges of $\mathrm{C}$ and $\mathrm{B}(\mathrm{N})$ at the $\mathrm{C}-\mathrm{B}(\mathrm{C}-\mathrm{N})$ interface. The bottom panel plots the change in the atomic charge at each interface.

\section{RESPONSE TO EXTERNAL ELECTRIC FIELDS}

We move on now to analyze the response of the interfacial electronic states to external electric fields, and explore the possible magnetoelectric effects. These calculations are performed for tubular geometries, under transversal electric fields applied along the carbon strip and perpendicular to the periodic tube axis, as shown in Fig. 1(b), hence avoiding the intrinsic difficulties to simulate electric fields under periodic boundary conditions (due to the intrinsic nonperiodic nature of the position operator [35]). In particular, we model $(8,8)$ armchair nanotubes with zigzag-edged $\mathrm{C} / \mathrm{BN}$ domains composed of $n=5$ zigzag chains of $\mathrm{C}$ and $m=11$ zigzag chains of $\mathrm{BN}$ along the tube axis, following the geometries discussed in the literature $[10,11,14,15,25]$. This chirality and chemical composition result in a half-metallic ground state [15]. An additional set of ghost orbitals ( $s$ and $p$ like) cylindrically distributed around the tube axis are used to improve the description of nearly free electron states known to exist in $\mathrm{BN}$ nanotubes [36].

Under no field, the spin Mulliken populations for $C_{B}$ and $C_{N}$ are $\sim 0.1 \mu_{B}$, which are aligned antiparallel at each interface. When an electric field is applied in the positive (negative) $x$ direction of the $\mathrm{C} / \mathrm{BN}$ nanotube [Fig. 1(b)], mobile electrons in the graphene ribbon are pushed towards the $\mathrm{B}$ edge $(\mathrm{N}$ edge), and depleted at the $\mathrm{N}$ edge ( $\mathrm{B}$ edge). The change in $\mathrm{C}_{\mathrm{B}}$ $\left(\mathrm{C}_{\mathrm{N}}\right)$ charge population increases (decreases) linearly with the external field strength (see the central panel in Fig. 6). This charge reorganization has a direct effect on the edge magnetic 


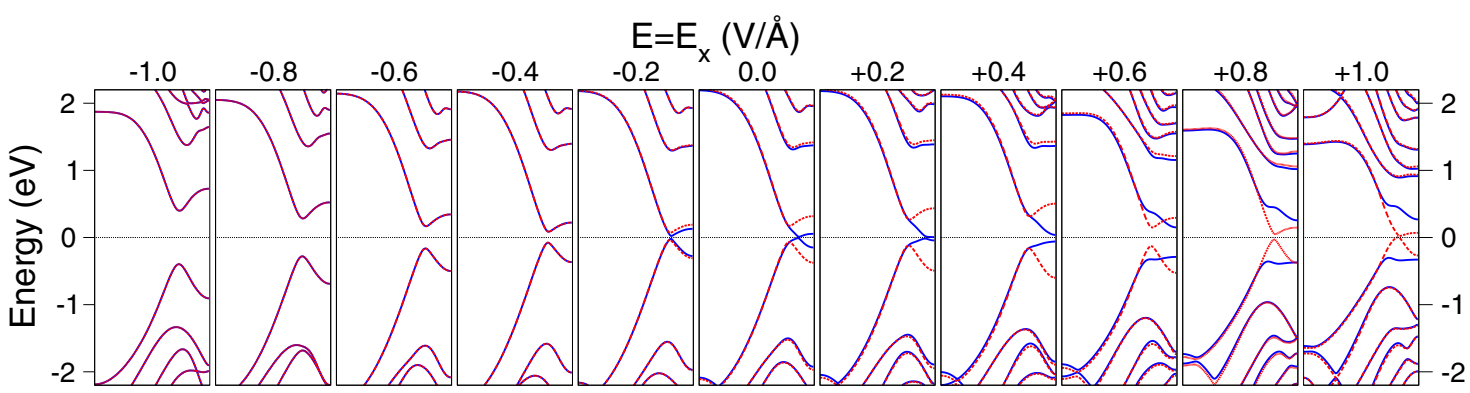

FIG. 7. (Color online) Band structure close to the Fermi level (horizontal dashed line) for the $\mathrm{C}_{5}(\mathrm{BN})_{11}$ hybrid nanotube under electric fields along the $x$ direction. Blue solid and red dotted lines correspond to $\beta$ and $\alpha$ spins, respectively. Energies are given in eV. Field strengths are given (in $\mathrm{V} / \AA$ ) on top of each panel.

properties [Fig. 4(c)]. Notice that for fields below $-0.3 \mathrm{~V} / \AA$, the edge magnetism is killed. The linear magnetoresistance at low field, defined as

$$
\alpha_{\mathrm{L}}=\mu_{0} \frac{\Delta M_{\mathrm{L}}}{E}
$$

(where $\Delta M_{\mathrm{L}}$ denotes the change in linear magnetization at the $\mathrm{C} / \mathrm{BN}$ interface), is four times larger than the value obtained for bilayer GNR on silicon substrates [26]. The bulk magnetoelectric coefficient (considering the volume of the whole hybrid $\mathrm{C} / \mathrm{BN}$ nanotube) would be $\sim 1 \mathrm{ps} / \mathrm{m}$, comparable to that of the prototypical magnetoelectric material $\mathrm{Cr}_{2} \mathrm{O}_{3}$. Notice that the dependence of the edge magnetism on the external electric field is completely different from that observed in graphene nanoribbons [26]. To understand this behavior the nature of the edge bands must be considered.

Figure 7 shows the band structure close to the Fermi level for both spin orientations, as a function of the electric field. An electric field pointing in the negative $x$ direction shifts the $\pi_{\mathrm{B}}$ valence band towards lower energies so that extra electrons populate the $\beta$-spin metallic states ( $\pi_{\mathrm{N}}^{*}$ moves up in energy and its $\beta$-spin metallic states are depopulated). Eventually, the $\pi_{\mathrm{B}} \beta$ band becomes completely filled (as was its $\alpha$-spin counterpart), while the $\pi_{\mathrm{N}}^{*}$ is emptied. When this happens (at fields below $-0.3 \mathrm{~V} \AA$ ) a gap is opened. Similar results have been reported recently by Liang and Kawazoe [37].

On the other hand, for electric fields pointing in the positive $x$ direction, the $\pi_{\mathrm{N}}^{*}$ band is stabilized, and conducting electrons ( $\beta$ spin) are transferred to the $\mathrm{N}$ edge from the $\mathrm{B}$ edge (the $\pi_{\mathrm{B}}$ band moves to higher energies), increasing the magnetization at the former interface. At approximately $+0.4 \mathrm{~V} / \AA$, a small gap opens. Larger electric fields move the $\alpha$ bands $\left(\pi_{\mathrm{B}}\right.$ up and $\pi_{\mathrm{N}}^{*}$ down) closer to the Fermi level, and for $E \geqslant 0.9 \mathrm{~V} / \AA$, the gap closes and the system again becomes half-semimetallic, with a spin inversion from the original state (the metallic bands are now $\alpha$ spin).

Although we have only studied the field response of CBN nanotubes with $(8,8)$ chirality, the effect of curvature on the electronic structure of the interfacial states is minor, and we can extrapolate the results to other geometries, including planar monolayers. In particular, similar magnetoelectric (ME) effects can be expected for hybrid CBN nanosheets when an in-plane electric field is applied perpendicular to the $\mathrm{C} / \mathrm{BN}$ interface. In this configuration, the piezoelectric response of $\mathrm{BN}$ and the edge-state spin polarization at the interfaces will be similar to those reported here for fields along the $x$ axis. Hence, the changes in the band structure and edge population are expected to give a surface ME coefficient comparable to that of GNR on silicon, though different in nature. Notice also that the surface ME constant for these half-metallic heterostructures would be two orders of magnitude larger than the universal constant predicted at the surface of bulk half-metals [38].

It has been shown that the relative composition and sizes of $\mathrm{C}$ and $\mathrm{BN}$ domains determine the band gaps of $\mathrm{C} / \mathrm{BN}$ heterostructures $[9,11,13,14,39]$. Very low concentrations of $\mathrm{C}$ give rise to semiconducting or insulating properties, while large concentrations suppress potential half-metallic properties. This, of course, will change the strengths of the threshold fields, but the ME effect will remain, and the results presented here will hold. Examples of the response of the interfacial charge to external electric fields for systems with chemical compositions are shown in Fig. 6. Interestingly, He et al. showed that half-metallicity could also be obtained when a few $\mathrm{C}$ zigzag chains (GNR) are attached to a $\mathrm{BN}$ nanoribbon (BNNR) [39]. Considering that the edge of the BN monolayers is relatively smooth, this could be an interesting path for the experimental realization of spintronic devices based in these hybrid $\mathrm{C} / \mathrm{BN}$ nanostructures.

\section{CONCLUSIONS}

In summary, we have shown different ways to engineer the electronic properties of hybrid C/BN nanostructures by tuning the bound charge at polar zigzag interfaces. By taking advantage of the dielectric properties in $\mathrm{BN}$ we can module half-metallicity by mechanical deformation, and our theoretical calculations predict a piezomagnetic effect in these hybrid systems. In addition, we have evaluated the response to external electric fields and obtained a strong magnetoelectric effect that could be of much interest for spintronic applications based on carbon nanostructures. We propose that the recently grown ultrathin $\mathrm{C}$ ribbons segregated from percolated $\mathrm{BC}_{2} \mathrm{~N}$ domains [33] could be candidates for the experimental observation of these effects. More importantly, the phenomena discussed here could also be expected for other two-dimensional materials 
whenever a polar discontinuity is present. These include grain boundaries in ionic monolayers (such as $\mathrm{BN}$ or transition metal dichalcogenides), and other hybrid systems, not necessarily with zigzag-shaped edges. Recently Gibertini and collaborators [22] showed that chemically modified honeycomb lattices can be engineered to give rise to polar discontinuities. Our work opens a route to fine tune the electronic reconstructions at one-dimensional polar interfaces in monolayered systems by taking advantage of their dielectric properties.

\section{ACKNOWLEDGMENTS}

We acknowledge support from the Spanish FEDERMINECO (Grant No. FIS2012-37549-C05-02) and Generalitat de Catalunya (2014 SGR 301 and AGAUR Grant No. FI-DGR 2011FI B 00993). We would like to thank the Spanish Supercomputer Network for computing resources. ICN2 acknowledges support of the Spanish MINECO through the Severo Ochoa Centers of Excellence Program under Grant No. SEV-2013-0295.
[1] S. Enouz, O. Stéphan, J.-L. Colliex, and A. Loiseau, Nano Lett. 7, 1856 (2007).

[2] L. Ci, L. Song, C. Jin, D. Jariwala, D. Wu, Y. Li, A. Srivastava, Z. F. Wang, K. Storr, L. Balicas et al., Nat. Mater. 9, 430 (2010).

[3] P. Sutter, R. Cortes, J. Lahiri, and E. Sutter, Nano Lett. 12, 4869 (2012).

[4] M. P. Levendorf, C.-J. Kim, L. Brown, P. Y. Huang, R. W. Havener, D. A. Muller, and J. Park, Nature (London) 488, 627 (2013).

[5] Y. Gao, Y. Zhang, P. Chen, Y. Li, M. Liu, T. Gao, D. Ma, Y. Chen, Z. Cheng, X. Qiu et al., Nano Lett. 13, 3439 (2013).

[6] R. Drost, A. Uppstu, F. Schulz, S. K. Hämäläinen, M. Ervasti, A. Harju, and P. Liljeroth, Nano Lett. 14, 5128 (2014).

[7] J. Lu, L. C. Gomes, R. W. Nunes, A. H. Castro-Neto, and K. P. Loh, Nano Lett. 14, 5133 (2014).

[8] M. Liu, Y. Li, P. Chen, J. Sun, D. Ma, Q. Li, T. Gao, Y. Gao, Z. Cheng, X. Qiu, Y. Fang, Y. Zhang, and Z. Liu, Nano Lett. 14, 6342 (2014).

[9] Y. Ding, Y. Wang, and J. Ni, Appl. Phys. Lett. 95, 123105 (2009).

[10] A. Du, Y. Chen, Z. Zhu, G. Lu, and S. C. Smith, J. Am. Chem. Soc. 131, 1682 (2009).

[11] Z.-Y. Zhang, Z. Zhang, and W. Guo, J. Phys. Chem. C 113, 13108 (2009).

[12] V. V. Ivanovskaya, A. Zobelli, O. Stéphan, P. R. Briddon, and C. Colliex, J. Phys. Chem. C 113, 16603 (2009).

[13] J. M. Pruneda, Phys. Rev. B 81, 161409(R) (2010).

[14] W. An and C. H. Turner, J. Phys. Chem. Lett. 1, 2269 (2010).

[15] J. M. Pruneda, Phys. Rev. B 85, 045422 (2012).

[16] M. Bernardi, M. Palummo, and J. C. Grossman, Phys. Rev. Lett. 108, 226805 (2012).

[17] I. I. Naumov and A. M. Bratkovsky, Phys. Rev. B 84, 245444 (2011).

[18] J. Qi, X. Qian, L. Qi, J. Feng, D. Shi, and J. Li, Nano Lett. 12, 1224 (2012).

[19] I. I. Naumov, A. M. Bratkovsky, and V. Ranjan, Phys. Rev. Lett. 102, 217601 (2009).

[20] K. H. Michel and B. Verberck, Phys. Rev. B 80, 224301 (2009).
[21] K.-A. N. Duerloo, M. T. Ong, and E. J. Reed, J. Phys. Chem. Lett. 3, 2871 (2012).

[22] M. Gibertini, G. Pizzi, and N. Marzari, Nat. Commun. 5, 5157 (2014).

[23] Y.-W. Son, M. L. Cohen, and S. G. Louie, Nature (London) 444, 347 (2006).

[24] S. Dutta, A. K. Manna, and S. K. Pati, Phys. Rev. Lett. 102, 096601 (2009).

[25] B. Huang, C. Si, H. Lee, L. Zhao, J. Wu, B.-L. Gu, and W. Duan, Appl. Phys. Lett. 97, 043115 (2010).

[26] Z. Zhang, C. Chen, and W. Guo, Phys. Rev. Lett. 103, 187204 (2009).

[27] J. Jung and A. H. MacDonald, Phys. Rev. B 81, 195408 (2010).

[28] J. P. Perdew, K. Burke, and M. Ernzerhof, Phys. Rev. Lett. 77, 3865 (1996).

[29] J. M. Soler, E. Artacho, J. D. Gale, A. García, J. Junquera, P. Ordejón, and D. Sánchez-Portal, J. Phys.: Condens. Matter 14, 2745 (2002).

[30] N. Troullier and J. L. Martins, Phys. Rev. B 43, 1993 (1991).

[31] N. C. Bristowe, M. Stengel, P. B. Littlewood, E. Artacho, and J. M. Pruneda, Phys. Rev. B 88, 161411 (2013).

[32] M. López-Suárez, M. Pruneda, G. Abadal, and R. Rurali, Nanotechnology 25, 175401 (2014).

[33] J. Lu, K. Zhang, X. F. Liu, H. Zhang, T. C. Sum, A. H. CastroNeto, and K. P. Loh, Nat. Commun. 4, 2681 (2013).

[34] A. Y. Liu, R. M. Wentzcovitch, and M. L. Cohen, Phys. Rev. B 39, 1760 (1989).

[35] I. Souza, J. Iñiguez, and D. Vanderbilt, Phys. Rev. Lett. 89, $117602(2002)$

[36] K. H. Khoo, M. S. C. Mazzoni, and S. G. Louie, Phys. Rev. B 69, 201401 (2004)

[37] Y. Liang and Y. Kawazoe, J. Chem. Phys. 140, 234702 (2014).

[38] C. G. Duan, C. W. Nan, S. S. Jaswal, and E. Y. Tsymbal, Phys. Rev. B 79, 140403(R) (2009).

[39] J. He, K.-Q. Chen, Z.-Q. Fan, L.-M. Tang, and W. P. Hu, Appl. Phys. Lett. 97, 193305 (2010). 